\title{
Multiple Fat- Containing Hepatic Hydatid Cysts: A Case Report in The X- Ray Institute at Medical City Health Directorate in Iraq
}

\author{
Issam Tariq Abdul-Wahaab $(\mathrm{PhD})^{1}$, Khaleel A Hadi $(\mathrm{ABHS}-\mathrm{Rad})^{2}$ and Haider \\ Abdulameer Ghayad (ABHS-Rad) ${ }^{3}$ \\ ${ }^{1}$ Department of anatomy,Faculty of Medicine, University of Baghdad, Baghdad, Iraq \\ ${ }^{2,3}$ X-ray Institute, Medical City Directorate, Baghdad, Iraq \\ Correspondence Address: \\ Issam Tariq Abdul-Wahaab \\ Department of anatomy,Faculty of Medicine, University of Baghdad, Baghdad, Iraq \\ email: dr.issam76t@gmail.com
}

Received: 23 May 2021

Accepted: 25 July 2021

Published: 25 December 2021

Diyala Journal of Medicine 2021:21(2): 64-69

\begin{abstract}
Background: Hydatidosis is a zoonotic disease caused by Echinococcus granulosus and Echinococcus multilocularis parasite which is still endemic in many countries all over the world especially in the developing countries. The liver is the primary site to be infested by the parasite with a rate of $60-75 \%$. The right lobe of the liver gets infected in about $80 \%$ of cases. Hydatid disease of the liver is usually asymptomatic and most cases discovered accidentally on routine clinical or radiological examinations for other illness. Symptoms usually appear in complicated cases either due to rupture, secondary bacterial infection, or due to the large size of the cyst which might cause pain in the right upper quadrant of the abdomen, discomfort, and sometimes swelling. In this case report, we reported the presence of fat globules within the hepatic hydatid cysts which is most probably due to rupture of hepatic hydatid cysts into the biliary tree.
\end{abstract}

Keywords: Hepatic, hydatid cyst, fat globule and CT scan

DOI: https://doi:10.26505/DJM.21026070530 , ( )Authors, 2021, College of Medicine, University of Diyala.

This is an open access article under the CC BY 4.0 license (http://creativecommons.org/licenses/by/4.0/)

\section{Introduction}

Hydatidosis is a zoonotic disease caused by Echinococcus granulosus and Echinococcus multilocularis parasite which is

still endemic in many countries all over the world especially in developing countries. The world health organization reported the 
incidence rate of hydatid disease exceeding more than 50 infections per 100000 individuals annually [1]. The liver is the primary site to be infested by the parasite with a rate of $60-75 \%$. The right lobe of the liver gets infected in about $80 \%$ of cases. Less commonly the parasite can infest the lungs, brain, spleen, bones, peritoneum, and kidneys. The cyst is composed of 3 lay outer, middle and inner layers. The outer layer is referred to as the pericyst which results as a defense mechanism from the tissue against the infestation. The inner or what is called the germinal layer produces the middle laminated membrane (middle layer) and the scolices. The middle and inner layers represent the endocyst [2]. Hydatid disease of the liver is usually asymptomatic and most cases are discovered accidentally on routine clinical or radiological examinations for other illnesses. Symptoms usually appear in complicated cases either due to rupture, secondary bacterial infection, or due to the large size of the cyst which might cause pain in the right upper quadrant of the abdomen, discomfort, and sometimes swelling [3]. Hydatid cysts might have a wide variety of imaging presentations at diagnosis such as simple cysts, cysts with multiple daughter cysts, calcifications whether intralesional or wall calcifications depending on the stage of the disease [4-6].

Complications of hepatic hydatid disease include: rupture into peritoneal or thoracic cavities and abscess formation due to secondary bacterial infection [7]. In addition to that hydatid cyst may rupture into the biliary tree [8]. The imaging modality of Hydatid disease represented by ultrasonography, computed tomography (CT) and magnetic resonant imagines (MRI). Ultrasonography is the most commonly used test to diagnose and evaluate hydatidosis of the liver; moreover, it plays an important role in the diagnosis of hydatid disease of spleen, kidneys, heart, pelvis and pleura. The typical ultrasonic finding for hepatic hydatid cyst is a well-circumscribed anechoic lesion that has a posterior acoustic enhancement with no signs of hepatic parenchymal infiltration. The lesion may show fine internal echoes which refer to hydatid sand and represent the debris. The mass effect may be evident when the size of the cyst is large enough to cause pressure on the surrounding structures [9]. Computed tomography and magnetic resonanceimaging have crucial roles in the assessment of extra-hepatic hydatidosis since they give more information about the site, size, and the extent of the lesion. The cyst appears as well-circumscribed hypoattenuating in $\mathrm{CT}$ examination and hypointense in $\mathrm{T} 1$ or hyperintense in $\mathrm{T} 2$ weight MR images. After parentral administration of contrast material, the hydatid cyst shows mild peripheral enhancement [10]. In this case report, we reported the presence of fat globule within the hepatic hydatid cyst which is most probably due to rupture of a hepatic hydatid cyst into the biliary tree.

\section{Case presentation}

A 39 year old female patient presented to the international center for medical specialization in Baghdad at the $22^{\text {nd }}$ of February 2017 complaining of right hypochondrial heaviness and discomfort for the last few months. She was referred to the ultrasonography unit for an abdominal ultrasound. The patient gave a history of 
previous medical visits and checkups from which she discovered the presence of multiple focal lesions in her liver which was thought to be of metastatic character. Abdominal ultrasound was performed for her to find out the lesions. In addition, CT scan was done for better characterization \& localization of the lesion $\&$ to exclude the possibility of malignancy.

\section{Radiological findings}

Abdominal ultrasonography was done by using GE Voluson 735 sonography which revealed the presence of multiple welldefined focal cystic and solid lesions in the right \& left lobes of the liver. The cystic lesions showed low-level internal echoes. The lesions were of different sizes and the largest was measuring $80 \mathrm{~mm}$ x $69 \mathrm{~mm}$ in segment VIII of the right lobe of the liver. CT scan was recommended to exclude metastasis. Thus the patient was examined by using Philips Brilliance 64 CT scanner. The patient was positioned quite supine on the $\mathrm{CT}$

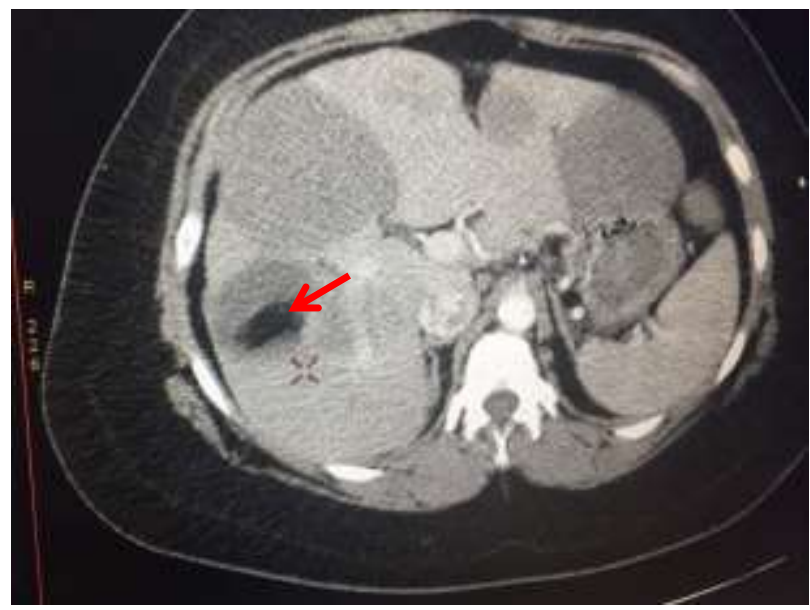

Axial non-contrast CT image shows well-defined cyst in segment VII of the liver with intra-cystic fat globules (red arrow) scan table. Non-contrast thin sections were carried out through the abdomen to detect any possible hepatic lesions. After that $80 \mathrm{ml}$ of non-ionic contrast material (Ultravist 370 and Omnipaque 350) was injected through a cannula placed in the right antecubital vein at a rate of $5.5 \mathrm{ml} / \mathrm{sec}$. CT scan examination revealed the presence of multiple rounded heterogeneous lesions in the right and left lobes of the liver namely in segment II, IVa, VII, and VIII, and the largest lesion located in segment VIII measuring approximately 77 $\mathrm{mm} \times 71 \mathrm{~mm}$. The lesions appeared to be hypodense with some of them containing fat density globules. Contrast-enhanced CT scan showed no obvious enhancing solid components. There was no evidence of intrahepatic biliary radical dilatation $\&$ the liver parenchyma looked normal. The picture was suggestive of multiple hepatic hydatid cysts.

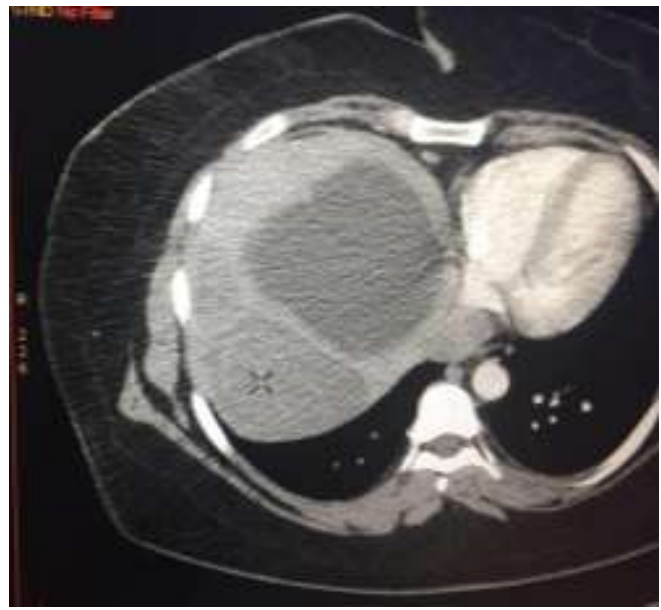

Axial CECT examination image showed no obvious enhancing solid components 

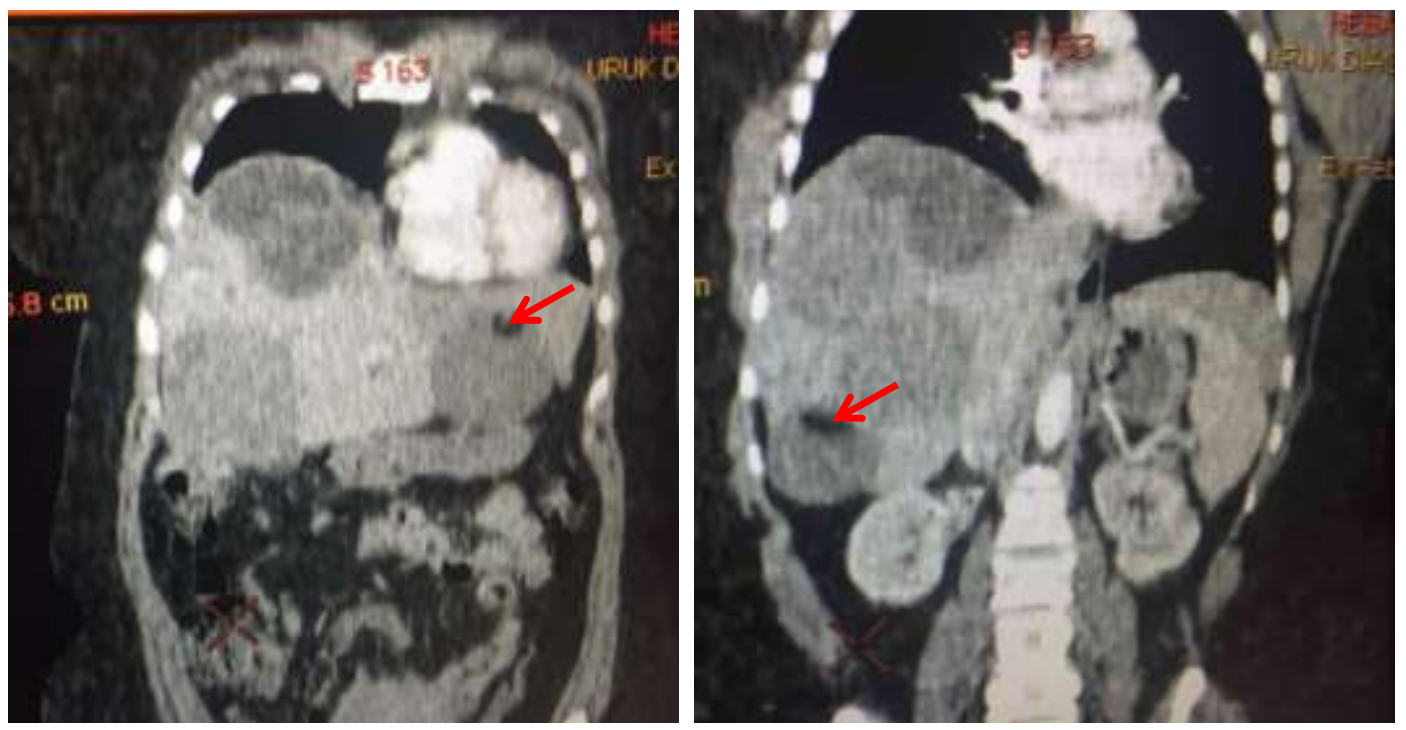

Coronal reformatted CT image showing multiple focal lesions in the right \& left lobes of the liver with intra-cystic fat globules (red arrow)

Differential Diagnosis: Complicated hepatic hydatid cyst, Hepatic angiomyolipoma and Hepatic teratoma.

Final Diagnosis: Diagnosis was certain by laparotomy and it was multiple hepatic hydatid cysts.

\section{Discussions}

Hepatic hydatid disease is caused by the larva of tapeworm, E. granulosus [2]. The radiological findings differ depending on the stage of the disease and the growth of the cyst which could be: unilocular simple cyst or complex large cyst with multiple daughter cysts in addition to the cystic wall calcification which could be partial or complete calcification [3]. Hepatic hydatid disease might be discovered incidentally as the patient might be asymptomatic or the patient presents with signs and symptoms due to the occurrence of the complications which could be infection or rupture. The modality of choice for diagnosis of hepatic hydatid disease is the CT scan examination which can detect the hypodense cystic lesion with well-circumscribed wall. The contrastenhancing CT scan reveals the rim enhancement of the wall with air-fluid level can be seen in cases of complications. Rupture of hepatic hydatid cyst occurs in about $20-50 \%$ of cases [13] and the main causes of the rupture are: 1) direct trauma, 2) degeneration of the cyst membranes and 3) rupture after reaching large size [2]. Rupture of hydatid cyst could be of three types:1) contained, 2) direct, and 3) communicating. In the first type the endocyst is ruptured while the pericyst remains intact with the membranes floating inside. Communicating rupture in which the cyst contents pass into the biliary tree. While when there is rupture for both endocyst and pericyst and spillage of the cyst content into the body cavities like pleural, peritoneal and pelvic cavities and into the hollow viscera of the abdomen is 
called the direct rupture. The presence of fat fluid level within the cyst is regarded as an indirect sign of communication rupture [7]. Previous studies like Mendez Montero et al. (1996) mentioned that the possible causes for the presence of fat globule within a hepatic hydatid cyst might be due to a cyst-biliary communication [7]. Malik et al. (2010) suggested that lipids play an important role in the metabolism of HHCs and that fat-fluid level in an HHC is related to degeneration of hydatid membranes (aging process of the cyst) [11]. The treatment of choice for complicated cases is surgical intervention [11]. While uncomplicated cases can be dealt with PAIR (puncture aspiration - injection respiration technique) which is a percutaneous ultrasonographic guided drainage technique with an injection of scolicidal agents [12]. Our case was diagnosed depending on the CT scan examination which is regarded as the modality of choice in diagnosing complicated cases. The presence of fat fluid level in the hepatic hydatid cyst, which is a rare condition, is indicative of the communication of the cyst with the biliary passages.

\section{Conclusions}

Hepatic hydatid cyst containing fat globules due to cystic rupture into the biliary tree.

\section{Recommendations}

It is important to keep in mind intra-biliary rupture of hepatic hydatid cysts.

Source of funding: This research was funded by ourselves and these is no other funding cover this study or manuscript preparation and publication.
Ethical clearance: The project was approved by the ethical committee and the institutional review board of Diyala College of Medicine in 2021.

\section{Conflict of interest: Nill}

\section{References}

[1]Greco S, Cannella R, Giambelluca D, Pecoraro G, Battaglia E, Midiri M, Brancatelli G, Vernuccio F. Complications of hepatic echinococcosis: multimodality imaging approach. Insights Imaging. 2019;10(1):113.

[2]Beggs I. The radiology of hydatid disease. AJR Am J Roentgenol. 1985;145(3):639648.

[3]Pedrosa I, Saíz A, Arrazola J, Ferreirós J, Pedrosa CS. Hydatid disease: radiologic and pathologic features and complications. Radiographics 2000;20(3):795-817.

[4]Eckert J, Gemmell MA, Meslin FX, Pawlowski ZS, World Health Organization. WHO/OIE manual on echinococcosis in humans and animals: a public health problem of global concern. Paris, France: World Organisation for Animal Health; 2001.

[5]Polat P, Kantarci M, Alper F, Suma S, Koruyucu MB, Okur A. Hydatid disease from head to toe. Radiographics 2003; 23(2):475-494; quiz 536-537.

[6]Bartolotta TV, Vernuccio F, Taibbi A, Lagalla R. Contrast-enhanced ultrasound in focal liver lesions: where do we stand? In Seminars in Ultrasound, CT and MRI 2016 Dec 1 (Vol. 37, No. 6, pp. 573-586). WB Saunders.

[7]Mendez Montero JV, Arrazola Garcia J, Lopez Lafuente J, Antela Lopez J, Mendez Fernandez R, Saiz Ayala A. Fat-fluid level in hepatic hydatid cyst: a new sign of rupture 
into the biliary tree? AJR Am J Roentgenol 1996;167(1):91-94.

[8]Marti-Bonmati L, Menor Serrano F. Complications of hepatic hydatid cysts: ultrasound, computed tomography, and magnetic resonance diagnosis. Gastrointest Radiol 1990 Spring;15(2):119-125.

[9]WHO Informal Working Group. International classification of ultrasound images in cystic echinococcosis for application in clinical and field epidemiological settings. Acta Trop. 2003;85(2):253-261.

[10]Graeter T, Kratzer W, Oeztuerk S, Haenle MM, Mason RA, Hillenbrand A, Kull T, Barth TF, Kern P, Gruener B. Proposal of a computed tomography classification for hepatic alveolar echinococcosis. World J Gastroenterol. 2016;22(13):3621-3631. [11]Malik AA, Bari SU, Amin R, Jan M. Surgical management of complicated hydatid cysts of the liver. World J Gastrointest Surg 2010;2(3):78-84.

[12]Zargar SA. Percutaneous drainage of liver hydatid cysts: is evidence enough to accept it as first modality of choice? Trop Gastroenterol.2011;32(3):161-163.

[13] De Diego Choliz J, Lecumberri Olaverri FJ, Franquet Casas T, Ostiz Zubieta S. Computed tomography in hepatic echinococcosis. AJR Am J Roentgenol 1982

Oct;139(4):699-702. 